\title{
CARACTERIZAÇÃO DOS ERROS ORTOGRÁFICOS EM CRIANÇAS COM TRANSTORNOS DE APRENDIZAGEM
}

\author{
Characterizing spelling mistakes found in children \\ with learning problems
}

Jaime Luiz Zorzi ${ }^{(1)}$, Sylvia Maria Ciasca ${ }^{(2)}$

\section{RESUMO}

Objetivos: analisar habilidades ortográficas de crianças diagnosticadas com problemas de aprendizado. Foram quantificados e classificados erros produzidos pelos sujeitos, visando descrever aqueles de maior freqüência, verificando se há diferença entre a ocorrência dos mesmos. Pretendeu-se caracterizar um perfil ortográfico indicativo das dificuldades gerais mais comumente encontradas nesta população. Métodos: foi examinada a escrita de 69 sujeitos avaliados pelo Laboratório de Distúrbios de Aprendizagem do Departamento de Neurologia da UNICAMP e diagnosticados como apresentando algum tipo de problema de aprendizagem. As idades variaram entre oito anos e dois meses e treze anos e quatro meses, com uma média de dez anos e seis meses. Foram incluídos somente sujeitos em nível alfabético de escrita, sem qualquer tipo de rebaixamento intelectual. Os erros encontrados foram classificados em onze categorias e quantificados para fins de análise estatística. Resultados: dez grupos foram diagnosticados, sendo o Transtorno do Déficit de Atenção o mais numeroso, seguido por Dificuldades Escolares e Transtornos Associados. A maior parte dos sujeitos cursava a terceira série do ensino fundamental. O tipo de erro mais recorrente foi "Representações Múltiplas", seguido de "Omissões" e "Apoio na Oralidade". Observou-se correlação significativa principalmente entre "Omissões" e "Outras Alterações". Conclusão: a maior dificuldade encontrada está centrada nas "Representações Múltiplas", revelando dificuldades principalmente no nível ortográfico. Os erros por omissão de letras decorrem de falhas no processo de análise fonológica e de correspondência fonema-grafema. A baixa ocorrência de erros por "Inversão" e por "Letras Parecidas", indica ausência de dificuldades de natureza visual-espacial.

DESCRITORES: Transtornos de Aprendizagem; Dislexia; Transtornos da Falta de Atenção com Hiperatividade

\section{INTRODUÇÃO}

O desenvolvimento de habilidades básicas para ler e escrever, graças ao seu impacto na educação recebe uma atenção especial, principalmente nas séries iniciais do ensino fundamental. Entretanto, tal aprendizagem pode ser um grande desafio para muitos e dificuldades variáveis podem surgir durante este processo. Neste sentido, um dos aspectos que chama a atenção, diz respeito à ortografia, isto é, ao

(1) Fonoaudiólogo; Professor do CEFAC - Saúde e Educação; Doutor em Educação pela Universidade Estadual de Campinas.

(2) Psicóloga; Professora da Universidade Estadual de Campinas; Doutora em Neurociências pela Universidade Estadual de Campinas. domínio da escrita convencional das palavras. Pode ser difícil, para muitas crianças, compreender como as palavras devem ser apropriadamente grafadas, o que pode ser observado nas alterações ortográficas presentes em suas produções escritas.

Tais fatos têm merecido a atenção de muitos pesquisadores gerando estudos comparativos sobre a complexidade ortográfica de diferentes línguas, o que pode torná-las mais ou menos transparentes e assim influenciar sua aprendizagem, facilitando-a ou dificultando-a ${ }^{1-4}$. Têm sido apontadas escritas com ortografias profundas e escritas com ortografias mais superficiais. $\mathrm{O}$ aspecto fundamental das diferenças reside na complexidade silábica e ortográfica. Na leitura, as estruturas silábicas afetariam principalmente a decodificação enquanto que 
a profundidade ortográfica afetaria a leitura tanto de palavras reais quanto de pseudopalavras ${ }^{5}$.

Uma atenção maior também tem sido dada às alterações ortográficas do português escrito no Brasil, com a finalidade de melhor compreendê-las ${ }^{6-13}$. Embora variem em termos de análises mais quantitativas ou qualitativas, de uma maneira uniforme, estes estudos indicam que os "erros" fazem parte da aprendizagem, podendo revelar hipóteses que as crianças vão, gradativamente, construindo para chegar a conhecimentos mais aprofundados sobre a escrita.

Desta forma, observa-se que as crianças cometem "erros" durante a aprendizagem da escrita até que, progressivamente, elas dominem de forma mais segura o sistema ortográfico. Conseqüentemente, os erros se tornam cada vez mais específicos e ocasionais. Porém, por outro lado, também se observa que algumas delas parecem ter uma trajetória diferente, exibindo uma diversidade e freqüência de alterações de escrita mais intensa e duradoura. Tais dificuldades podem, além de revelarem uma possível má qualidade de ensino, ser sintoma de problemas ou limitações, como os distúrbios de aprendizagem e as dislexias 1,3,11,12,14-21. Muitas destas crianças acabam sendo encaminhadas para profissionais especializados para diagnóstico e atendimento extra-escolar.

A leitura e a escrita envolvem o uso de estratégias fonológicas e ortográficas sendo que algumas crianças podem ter grandes dificuldades com os aspectos fonológicos enquanto que outras podem ter tais limitações quanto aos aspectos ortográficos. Poderia haver uma influência genética mais forte sobre as dificuldades fonológicas, enquanto que os déficits de natureza ortográfica teriam maior influência ambiental, dado seu caráter convencional ${ }^{22}$. Têm sido apontadas dificuldades na representação da estrutura fonológica em palavras mesmo em disléxicos aprendendo a ortografia de escritas consideras como mais transparentes ${ }^{1}$. Neste sentido, a dislexia do desenvolvimento estaria mais relacionada aos déficits de decodificação de origem fonológica. Por sua vez, os déficits ortográficos estariam ligados a um atraso mais geral do desenvolvimento da linguagem, determinado por insuficiente exposição da criança à linguagem escrita ${ }^{22}$.

A existência de aspectos fonológicos e ortográficos determinando a escrita das palavras pode levar a diversos tipos de erros os quais também podem ter freqüências distintas de ocorrência. Esta incidência variável reforça a hipótese de que a presença destes desvios pode ser influenciada por características intrínsecas da própria linguagem escrita as quais demandam diferentes habilidades ou conhecimentos por parte do aprendiz ${ }^{7,11,23-27}$. Na ortografia do inglês, por exemplo, fatores como freqüência e comprimento das palavras, assim como os modos de combinação das letras têm sido apontados como aspectos associados às dificuldades na soletração e na leitura, ao ponto de levar autores ao questionamento se não seria o inglês escrito, em si, uma "língua disléxica" ${ }^{24}$.

A aprendizagem depende de condições estruturais e funcionais do sistema nervoso central. Disfunções que ocorram nas etapas de desenvolvimento das unidades funcionais cerebrais podem acarretar alterações na linguagem e na aprendizagem. Problemas de atenção e concentração são derivados de disfunções nas zonas primárias. Dificuldades na aprendizagem envolvendo a leitura, a escrita e a matemática podem ser derivadas de disfunções nas zonas secundárias. Por sua vez, o baixo rendimento cognitivo, falhas na compreensão da linguagem falada, limitações em termos de leitura escrita e matemática, estão relacionadas a disfunções nas áreas terciárias ${ }^{28}$.

Mais especificamente, existem evidências de que os diferentes tipos de dislexia podem estar associados a diferentes disfunções de áreas corticais específicas: a dislexia fonológica resultaria de disfunções no lobo temporal; a dislexia diseidética estaria associada a disfunções do lobo occipital e a dislexia mista a alterações funcionais do lobo préfrontal, frontal, occipital e temporal ${ }^{28}$. Tais achados têm sido alvos de muitas investigações graças aos avanços dos exames por meio de neuroimagens, particularmente as imagens obtidas por meio da ressonância magnética funcional, a fim de se estudar a ativação cerebral durante o processamento de linguagem, em condições variadas de apresentação de estímulos. Diferenças no funcionamento cerebral, nas áreas envolvidas no processamento de linguagem de crianças apresentando problemas de leitura e escrita têm sido confirmadas ${ }^{29}$.

Deve-se considerar que a apropriação do sistema de escrita é um processo evolutivo no qual o aprendiz vai elaborando hipóteses ou idéias a respeito do que é a escrita, as quais revelam diferentes graus de conhecimentos que estão sendo constituídos. Isto significa que não se aprende a escrever de imediato e que "erros" estão implícitos em tal processo ${ }^{30}$.

Porém, a permanência de erros pode refletir déficits que produzem dificuldades ou lentidão acentuada no sentido de a criança gerar e generalizar hipóteses que, sucessivamente, permitam a apreensão cada vez mais aprofundada da escrita. Tais crianças estabilizariam hipóteses elementares e insuficientes a respeito da ortografia. Uma série de conhecimentos apontados como fundamentais, principalmente a precisão das representações 
fonológicas e o conhecimento das convenções ortográficas, não estariam sendo adequadamente desenvolvidos, provocando as dificuldades na escrita das palavras ${ }^{10,31,32}$.

Os diversos estudos sobre dificuldades em leitura, assim como em soletração e ortografia têm sido feitos principalmente com crianças disléxicas $1,2,16,23,33,34$. Por outro lado, pouca atenção tem sido dada a outros tipos de problemas, como os distúrbios específicos de linguagem ${ }^{17,35}$. Pode-se também apontar a escassez de pesquisas envolvendo os problemas ortográficos nos distúrbios de aprendizagem, déficits de atenção e outros transtornos que comumente são encaminhados para diagnóstico e intervenção.

Uma das questões que merece investigação mais aprofundada diz respeito à análise dos tipos de erros mais comumente encontrados nas crianças apresentando transtornos de aprendizagem, assim como suas freqüências de ocorrência. Poderiam tais erros indicar prováveis déficits subjacentes, ligados às representações fonológicas ou ao conhecimento das convenções ortográficas? Haveria uma área mais prejudicada do que a outra, em termos de um perfil geral? Enfim, o que estes erros podem indicar em termos de limitações em algumas das habilidades fundamentais para o aprendizado da escrita?

Considerando-se que os erros ortográficos estão presentes em muitas crianças apresentando diversos tipos de problemas de aprendizagem e não unicamente na dislexia, o objetivo desta pesquisa é o de analisar a produção escrita de sujeitos classificados nestas condições. Ou seja, a partir de escritos de grupos de crianças diagnosticadas como tendo alguma limitação em termos de aprendizado da ortografia, serão quantificados e classificados os erros encontrados tendo em vista descrever aqueles de maior freqüência, verificando se há diferença entre a ocorrência dos mesmos. Desta forma, pretende-se caracterizar um perfil ortográfico que possa ser indicativo das dificuldades gerais mais comumente encontradas nestes grupos com transtornos de aprendizagem. De modo mais específico, os seguintes objetivos dirigem este trabalho: traçar o perfil ortográfico de escolares com diversos tipos de problemas de aprendizagem; verificar se os principais tipos de erros produzidos são os mesmos encontrados na aprendizagem considerada normal; verificar, na amostra, possíveis diferenças em termos de freqüência de ocorrência dos diversos tipos de erros; e verificar os possíveis déficits subjacentes a partir dos tipos de erros e de suas freqüências de ocorrência.

\section{MÉTODOS}

Faz parte desta pesquisa um grupo de 69 sujeitos que passaram pelo processo diagnóstico do Laboratório de Distúrbios de Aprendizagem do Departamento de Neurologia da Faculdade de Ciências Médicas da Universidade Estadual de Campinas (DISAPRE) e que foram diagnosticados como apresentando algum tipo de problema de aprendizagem. As idades variaram entre oito anos e dois meses e treze anos e quatro meses, com uma média de dez anos e seis meses. Tais sujeitos, de ambos os sexos, que freqüentam desde a primeira série do ensino fundamental até o segundo ano do ensino médio de escolas públicas e particulares, chegam com a queixa de problemas na aprendizagem para serem avaliados pela equipe multiprofissional do Laboratório tendo em vista o diagnóstico diferencial e encaminhamentos: avaliação neurológica, neuropsicológica e fonoaudiológica. Foram incluídos somente sujeitos que já haviam alcançado a fase alfabética de aquisição da escrita e que não apresentavam qualquer tipo de rebaixamento intelectual. Um dos participantes não apresentou respostas em todas as variáveis e por isso, ora a amostra é de 69 ora de 68 sujeitos. A amostra ficou caracterizada da seguinte maneira: Transtorno do Déficit de Atenção/Hiperatividade (TDA/H); Dificuldades de Aprendizagem; Distúrbio de Aprendizagem; Dislexia; Disgrafia; Altas Habilidades; Dificuldades Ortográficas; Síndrome de Prader-Willis; Distúrbios Associados e Diagnóstico não Conclusivo.

O procedimento para coleta do material escrito está composto de duas partes: um ditado de palavras reais e inventadas, previamente elaborado para medir uma série de conhecimentos ortográficos, e de uma redação a partir de um tema dado ("Uma casa abandonada"), por meio da qual é verificado o nível de domínio da ortografia em situação de produção de escrita espontânea. O material escrito foi coletado durante o processo de avaliação/diagnóstico por um avaliador previamente orientado quanto à forma de propor a situação para obtenção dos dados. Os erros foram analisados de acordo com os critérios de Zorzi ${ }^{9}$.

Compõem a pesquisa os sujeitos cujos responsáveis permitiram, por escrito, sua participação a partir dos esclarecimentos contidos no Termo de Consentimento Livre e Esclarecido. Este projeto foi aprovado no Comitê de Ética e Pesquisa do CEFAC - Pós Graduação em Saúde e Educação, tendo sido aprovado e registrado sob o $\mathrm{n}^{\circ}$ 025/08.

O tratamento estatístico foi realizado a partir do emprego dos seguintes testes: Wilcoxon, para comparar os resultados entre o principal tipo de erro e os demais; Igualdade de Duas Proporções, para 
comparar os percentuais de ocorrência entre todos os tipos de problemas; Correlação de Spearman para medir o grau de relação entre todos os tipos de erros; Teste de Correlação, para validação das correlações e o Intervalo de Confiança para Média. O nível de significância adotado foi de 5\%.

\section{RESULTADOS}

A Tabela 1 caracteriza a distribuição dos tipos de problemas de aprendizagem apresentados pelos sujeitos. O grupo diagnosticado como Transtorno do Déficit de Atenção e Hiperatividade (TDA/H) é o mais numeroso, denotando uma diferença estatisticamente significante em relação a todos os demais.

Tabela 1 - Distribuição dos tipos de problemas de aprendizagem

\begin{tabular}{lccc}
\hline Problemas & $\begin{array}{r}\text { Qtde } \\
\mathbf{N}=68\end{array}$ & $\%$ & p-valor \\
\hline TDA/H & 27 & $39,1 \%$ & \\
$\begin{array}{l}\text { Dificuldade escolar } \\
\text { Diagnóstico não }\end{array}$ & 13 & $18,8 \%$ & $0,009^{*}$ \\
$\begin{array}{l}\text { conclusivo } \\
\text { Distúrbio de }\end{array}$ & 8 & $11,6 \%$ & $<0,001^{*}$ \\
aprendizagem & 7 & $10,1 \%$ & $<0,001^{*}$ \\
Associados & 6 & $8,7 \%$ & $<0,001^{*}$ \\
$\begin{array}{l}\text { Dislexia } \\
\text { Altas habilidades }\end{array}$ & 3 & $4,3 \%$ & $<0,001^{*}$ \\
$\begin{array}{l}\text { Dificuldade } \\
\text { ortográfica }\end{array}$ & 1 & $1,4 \%$ & $<0,001^{*}$ \\
$\begin{array}{l}\text { Disgrafia } \\
\text { Síndrome de }\end{array}$ & 1 & $1,4 \%$ & $<0,001^{*}$ \\
Prader-Willis & 1 & $1,4 \%$ & $<0,001^{*}$ \\
\hline
\end{tabular}

Conforme os dados da Tabela 2, a maior parte dos sujeitos estava freqüentando desde a segunda até a quinta séries do ensino fundamental, com uma concentração maior na terceira série. Somente um deles estava cursando o segundo ano do ensino médio. Não se obteve informação precisa a respeito do grau de escolaridade de 4 sujeitos.

A Tabela 3 descreve os diversos tipos de erros e apresenta uma análise completa da ocorrência dos mesmos, considerando-se todos os sujeitos. O tipo de erro com maior resultado foi Representações Múltiplas, com média de 17,14 $\pm 2,04$ de intervalo de confiança. A análise dos $p$-valores indica que este é o tipo de erro mais recorrente, com uma diferença significante em relação aos demais. Em

Tabela 2 - Distribuição dos sujeitos por grau de escolaridade

\begin{tabular}{|c|c|c|}
\hline Escolaridade & Total & Porcentagem \\
\hline $1^{\text {a }}$ série & 2 & $2,89 \%$ \\
\hline $2^{\mathrm{a}}$ série & 12 & $17,39 \%$ \\
\hline $3^{a}$ série & 19 & $27,53 \%$ \\
\hline $4^{a}$ série & 12 & $17,39 \%$ \\
\hline $5^{\mathrm{a}}$ série & 12 & $17,39 \%$ \\
\hline 6aㅗ série & 7 & $10,14 \%$ \\
\hline $7^{a}$ série & 0 & $0 \%$ \\
\hline 8ª série & 0 & $0 \%$ \\
\hline $1^{\circ}$ colegial & 0 & $0 \%$ \\
\hline $2^{\circ}$ colegial & 1 & $1 \%$ \\
\hline $3^{\circ}$ colegial & 0 & $0 \%$ \\
\hline desconhecida & 4 & $5,79 \%$ \\
\hline Total & 69 & $100,00 \%$ \\
\hline
\end{tabular}

Tabela 3 - Descrição dos tipos de erros e média de ocorrência

\begin{tabular}{|c|c|c|c|c|c|c|c|c|c|c|c|}
\hline Tipos de Erros & Média & Mediana & $\begin{array}{l}\text { Desvio } \\
\text { Padrão }\end{array}$ & CV & Q1 & Q3 & Min & Max & $\mathbf{N}$ & IC & n-valor \\
\hline $\begin{array}{l}\text { representações } \\
\text { múltiplas }\end{array}$ & 17,14 & 18 & 8,63 & $50,3 \%$ & 11 & 23 & 0 & 35 & 69 & 2,04 & $p$-valui \\
\hline apoio na oralidade & 7,57 & 6 & 5,87 & $77,6 \%$ & 3 & 12 & 0 & 25 & 69 & 1,39 & $<0,001^{*}$ \\
\hline omissão de letras & 10,85 & 5 & 13,63 & $126 \%$ & 2 & 11,5 & 0 & 48 & 68 & 3,24 & $<0,001^{*}$ \\
\hline junção/separação & 2,49 & 2 & 2,87 & $115 \%$ & 0 & 4 & 0 & 12 & 69 & 0,68 & $<0,001^{*}$ \\
\hline am / ão & 2,09 & 1 & 3,28 & $157 \%$ & 0 & 3 & 0 & 21 & 69 & 0,77 & $<0,001^{*}$ \\
\hline generalização & 1,25 & 1 & 1,43 & $115 \%$ & 0 & 2 & 0 & 6 & 69 & 0,34 & $<0,001^{*}$ \\
\hline $\begin{array}{l}\text { Trocas } \\
\text { surdas/sonoras }\end{array}$ & 2,97 & 1 & 5,29 & $178 \%$ & 0 & 3 & 0 & 26 & 69 & 1,25 & $<0,001^{*}$ \\
\hline $\begin{array}{l}\text { Acréscimo de } \\
\text { letras }\end{array}$ & 1,77 & 1 & 2,14 & $121 \%$ & 0 & 2 & 0 & 9 & 69 & 0,51 & $<0,001^{*}$ \\
\hline letras parecidas & 0,90 & 0 & 2,09 & $232 \%$ & 0 & 1 & 0 & 15 & 69 & 0,49 & $<0,001^{*}$ \\
\hline inversões & 0,72 & 0 & 1,66 & $229 \%$ & 0 & 1 & 0 & 10 & 69 & 0,39 & $<0,001^{*}$ \\
\hline outras & 5,46 & 2 & 7,69 & $141 \%$ & 0 & 9 & 0 & 31 & 69 & 1,81 & $<0,001^{*}$ \\
\hline
\end{tabular}

Legenda: CV - coeficiente de variação; Q1 - 1ำ quartil; Q3 - 3ํo quartil; Min - mínimo; Max - máximo; IC - Intervalo de confiança 
segundo lugar vêm os erros por Omissão de Letras, seguido pelos erros decorrentes de Apoio na Oralidade. Na seqüência, com uma tendência de queda para médias mais reduzidas vêm os demais tipos de erros, com as Inversões de Letras na última posição, dada a baixa presença deste tipo de erro.

Possíveis correlações entre os diversos tipos de erros, assim como o grau que eles possam ter, estão expostas nas Tabelas 4 e 5, a primeira mostrando as correlações e a segunda, os p-valores. Constata-se que existem diversas correlações significantes. A mais forte encontrada foi entre os erros "Omissão de Letras" e "Outras", com uma correlação de 69,6\% ( $p<0,001)$. A segunda maior correlação ocorreu entre "Omissão" e "Apoio na Oralidade", com $64,0 \%(p<0,001)$. Seguem as correlações entre "Junção/separação" e "Representações Múltiplas", com $61,1 \%(p<0,001)$ e entre "Apoio na Oralidade" e "Outras", com 55,4\% ( $p<0,001)$. Toda correlação significante é positiva indicando que, conforme aumenta um dos erros, o outro tende a aumentar também e vice-versa. Deve-se ainda considerar o grau da correlação, que tende a ser mais intenso acima dos $60 \%$.

A Figura 1 exibe o número médio de cada tipo de erro e os intervalos de confiança para cada um deles. Tais intervalos, ora somados e ora subtraídos da média, indicam a variação da média segundo uma probabilidade estatística.

\section{Tabela 4 - Correlação dos tipos de erros}

\begin{tabular}{|c|c|c|c|c|c|c|c|c|c|c|}
\hline Correlação & $\begin{array}{l}\text { representações } \\
\text { múltiplas }\end{array}$ & oralidade & omissão & junção/sep. & am / ão & general. & sur/son. & acréscimo & $\begin{array}{l}\text { letras } \\
\text { par. }\end{array}$ & inversões \\
\hline oralidade & $36,1 \%$ & & & & & & & & & \\
\hline omissão & $39,5 \%$ & $64,0 \%$ & & & & & & & & \\
\hline junção/sep & $61,1 \%$ & $22,1 \%$ & $20,7 \%$ & & & & & & & \\
\hline am / ão & $39,7 \%$ & $23,9 \%$ & $11,6 \%$ & $23,6 \%$ & & & & & & \\
\hline general. & $12,6 \%$ & $1,0 \%$ & $-12,7 \%$ & $6,8 \%$ & $11,8 \%$ & & & & & \\
\hline sur/son. & $37,3 \%$ & $35,3 \%$ & $39,3 \%$ & $15,9 \%$ & $17,8 \%$ & $-19,8 \%$ & & & & \\
\hline acréscimo & $29,8 \%$ & $42,9 \%$ & $43,6 \%$ & $28,0 \%$ & $6,3 \%$ & $-4,9 \%$ & $23,0 \%$ & & & \\
\hline letras par. & $42,2 \%$ & $25,0 \%$ & $30,1 \%$ & $12,8 \%$ & $29,4 \%$ & $-3,7 \%$ & $22,1 \%$ & $27,6 \%$ & & \\
\hline inversões & $26,8 \%$ & $26,3 \%$ & $44,5 \%$ & $13,7 \%$ & $19,1 \%$ & $-2,1 \%$ & $28,2 \%$ & $48,8 \%$ & $30,5 \%$ & \\
\hline outras & $48,1 \%$ & $55,4 \%$ & $69,6 \%$ & $15,3 \%$ & $11,9 \%$ & $-4,8 \%$ & $44,0 \%$ & $52,1 \%$ & $43,5 \%$ & $43,8 \%$ \\
\hline
\end{tabular}

Tabela 5 - P-valores das correlação dos tipos de erros

\begin{tabular}{|c|c|c|c|c|c|c|c|c|c|c|}
\hline P-valor & $\begin{array}{c}\text { representações } \\
\text { múltiplas }\end{array}$ & oralidade & omissão & junção/sep. & $\begin{array}{c}\text { am / } \\
\text { ão }\end{array}$ & general. & sur/son. & acréscimo & $\begin{array}{l}\text { letras } \\
\text { par. }\end{array}$ & inversões \\
\hline oralidade & $0,002^{*}$ & & & & & & & & & \\
\hline omissão & $0,001^{*}$ & $<0,001^{*}$ & & & & & & & & \\
\hline junção/sep. & $<0,001^{*}$ & $0,068 \#$ & 0,090\# & & & & & & & \\
\hline am / ão & $0,001^{*}$ & $0,048^{*}$ & 0,347 & $0,051 \#$ & & & & & & \\
\hline general. & 0,303 & 0,932 & 0,302 & 0,577 & 0,335 & & & & & \\
\hline sur/son. & $0,002^{*}$ & $0,003^{\star}$ & $0,001^{*}$ & 0,193 & 0,144 & 0,104 & & & & \\
\hline acréscimo & $0,013^{*}$ & $<0,001^{*}$ & $<0,001^{*}$ & $0,020^{*}$ & 0,609 & 0,692 & $0,058 \#$ & & & \\
\hline letras par. & $<0,001^{*}$ & $0,038^{*}$ & $0,012^{*}$ & 0,294 & $0,014^{*}$ & 0,762 & $0,067 \#$ & $0,022^{*}$ & & \\
\hline inversões & $0,026^{*}$ & $0,029^{*}$ & $<0,001^{*}$ & 0,262 & 0,116 & 0,863 & $0,019^{*}$ & $<0,001^{*}$ & $0,011^{*}$ & \\
\hline outras & $<0,001^{*}$ & $<0,001^{*}$ & $<0,001^{*}$ & 0,208 & 0,329 & 0,698 & $<0,001^{*}$ & $<0,001^{*}$ & $<0,001^{*}$ & $<0,001^{*}$ \\
\hline
\end{tabular}

\section{DISCUSSÃO}

Dentre o grupo de sujeitos avaliados (Tabela 1), observam-se nove categorias de problemas. Chama a atenção o grande número de crianças com TDA/H, significativamente maior que todos os demais. Juntamente com os problemas de Dificuldades Escolares, Diagnósticos não conclusivos, Distúrbios de Aprendizagem e Distúrbios Associados, as crianças assim diagnosticadas compõem $88,3 \%$ da amostra. Por outro lado, constata-se um pequeno número de sujeitos que receberam o diagnóstico de Dislexia, assim como a ocorrência única de casos de Altas Habilidades, Dificuldade Ortográfica isolada, Disgrafia e Síndrome de Prader-Willis. Esta distribuição, de um modo geral, pode ser indicativa dos tipos de problemas mais comumente encontrados nas escolas e que motivam encaminhamentos.

A distribuição dos sujeitos, por grau de escolaridade (Tabela 2) revela que o encaminhamento escolar de crianças com problemas de aprendizagem tende a aumentar de acordo com o correr 


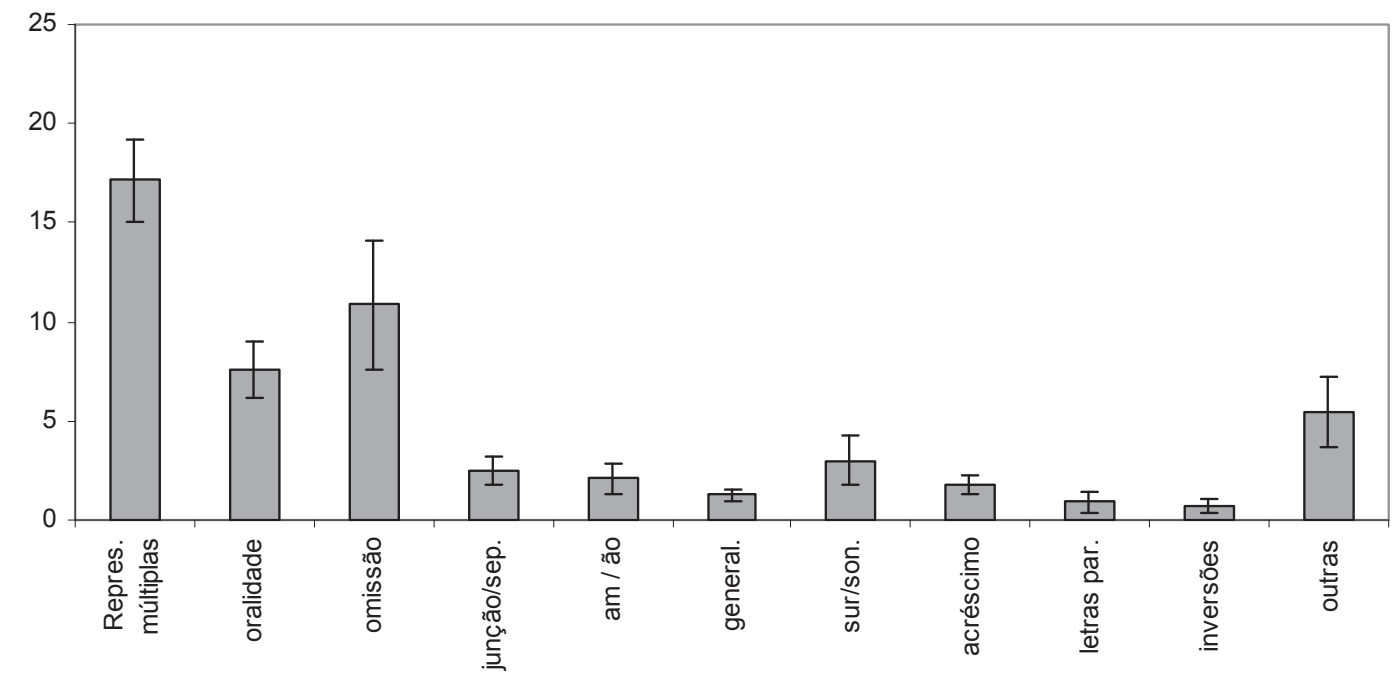

Figura 1 - Intervalo de confiança para os tipos de erros

dos anos escolares, atingindo um pico na $3^{\text {a }}$ série e decaindo a partir da $6^{\mathrm{a}}$ série. Considerados no conjunto, os sujeitos encaminhados cursando da $2^{\text {a }}$ até a $5^{\underline{a}}$ séries, compõem $79,67 \%$ da amostra. Poucas crianças são encaminhadas já na 1르 (somente $2,89 \%$ ) o que pode denotar uma atitude de espera ou expectativa, sem procedimentos de identificação e encaminhamentos o mais prontamente possível tendo sido detectada alguma dificuldade mais acentuada no desempenho do aluno. Este fato parece ser confirmado pelo aumento progressivo dos encaminhamentos, com maior volume na $3^{\underline{a}}$ série. Por outro lado, o fato de os encaminhamentos diminuírem de modo significativo a partir da $6^{\underline{a}}$ série pode ser indicativo de que não há uma preocupação pedagógica em termos de atendimento especializado aos alunos das séries mais avançadas. Estes dados também podem ser sugestivos de que os alunos que vêm apresentando problemas de aprendizagem ao longo das séries iniciais sejam aqueles que acabam abandonando a escola, tendo em vista a situação persistente de baixo desempenho ou de fracasso escolar.

Dentre todos os erros cometidos pelo conjunto de sujeitos, independentemente do tipo de problema apresentado (Tabela 3), os mais recorrentes, com uma diferença estatisticamente significante em relação aos demais, foram as "Representações Múltiplas". Entram nesta categoria aquelas alterações ortográficas que não decorrem de uma falha ou de uma inadequação nas correspondências entre fonemas e grafemas. Tais questões são de outra ordem, ligadas ao fato de que um mesmo fonema pode ter várias letras para representá-lo, assim como uma mesma letra pode representar vários fonemas. Nestes casos, entram em jogo uma série de variáveis que podem definir qual a opção correta para a palavra, incluindo regras contextuais, gramaticais ou mesmo etimológicas. Por exemplo, tanto a letra "m" quanto a letra " $n$ " podem ser usadas para se escrever o final de sílabas contendo vogais nasais (am/an; em/en; im/in; om/on e um/ um), como em "ponte" e "campo". Porém, o uso de uma ou de outra destas letras está subordinado a uma regra contextual que define qual das duas é a correta, dependendo da primeira letra da sílaba seguinte, o que gera a regra "Antes de $p$ e $b$ usa-se $m$ ". Da mesma forma, os conflitos entre o uso de "r" ou "rr", "x" ou "ch", "j" ou "g", do valor sonoro da letra "c" (se /k/ ou /s/), entram nesta categoria 9,15. Em síntese, a grafia correta de tais palavras vai além da compreensão de sua composição sonora, ou seja, de habilidades fonológicas, entrando na dimensão de questões ortográficas mais complexas.

Os resultados evidenciam que a maior parte dos erros produzidos pelos sujeitos da pesquisa diz respeito a um conhecimento ainda não suficientemente consolidado a respeito das características ortográficas do português o que provoca um grande número de substituição de letras as quais, embora possam escrever o fonema em questão, não são apropriadas para as palavras que estão sendo grafadas (churrasco > churasco; caçador > casador; serviço > cerviso). Déficits desta natureza têm sido apontados como decorrentes de atrasos mais gerais do desenvolvimento da linguagem e a fatores escolares, revelando uma insuficiente exposição dos sujeitos às características ortográficas da linguagem escrita ${ }^{22}$. Deve-se considerar que este é o tipo de erro também mais presente nos escritos de crianças sem queixas de aprendizagem, confirmando ser uma área de grande complexidade de 


\section{Roteiro de observação ortográfica}

Zorzi JL, 2006

\section{INTRUÇÕES PARA USO DO ROTEIRO DE OBSERVAÇÃO ORTOGRÁFICA}

Ditados

1. As listas de palavras foram elaboradas com a finalidade de garantir certas ocorrências ortográficas do português.

2. As palavras devem ser lidas de modo natural e claramente, isto é, da maneira como são pronunciadas normalmente nas conversações espontâneas. Não forçar a pronúncia artificial das palavras, ou seja, não produzir a palavra da forma como se escreve quando esta é diferente da forma natural de falar (ex: "tesoura" é o modo de escrever, enquanto "tisora" é o modo de falar).

3. Não dar nenhuma pista para as crianças a respeito da maneira como as palavras devem ser escritas. O objetivo é verificar como as crianças, por si mesmas, acreditam ser o modo de escrever as palavras apresentadas. Não se preocupem caso elas façam erros. São os processos que levam aos erros e acertos que queremos examinar.

\section{A. Ditado de palavras}

\begin{tabular}{|l|l|l|l|}
\hline Caçador & Carroça & Explicação & ovelha \\
\hline Assaltou & Presente & Travesseiro & Zelador \\
\hline Churrasco & Cimento & Vizinho & Enxugar \\
\hline Queixo & Machucado & Manchar & Gelatina \\
\hline Tijolo & Bandeja & Girassol & Jornal \\
\hline Comeram & Compraram & Viajaram & Falaram \\
\hline Soltou & Quente & Guerra & Tanque \\
\hline Mangueira & Sangue & Queimar & Macarrão \\
\hline combinar & Serviço & Cantaram & Estudam \\
\hline Exemplo & Quatrocentos & bombeiro & folha \\
\hline
\end{tabular}

\section{B. Ditado de pseudopalavras}

\begin{tabular}{|l|l|l|l|l|}
\hline langesa & guiçal & guice & sorama & naceso \\
\hline loprão & socraram & cortel & exampe & estongue \\
\hline jaram & gurra & musquera & enraiver & sompa \\
\hline pranço & esoge & zetrão & aspitra & Quesagilam \\
\hline
\end{tabular}

Figura 2 - Roteiro de observação ortográfica

conhecimentos, como é o caso da aprendizagem de regras contextuais e de regras morfossintáticas. Tais alterações, mais freqüentes nas séries iniciais, tendem a diminuir gradativamente, evidenciando que progressivamente as crianças vão se apropriando das regras ortográficas e estabilizando a forma convencional de escrever as palavras ${ }^{7,10}$. Este dado, por sua vez, reforça a hipótese da forte influência das condições de ensino e da maior experiência com a linguagem escrita sobre o domínio da ortografia.

O segundo maior conjunto de erros pertence à categoria da "omissão de letras". As omissões podem ocorrer por razões variadas. Uma delas pode ser devida a uma análise ainda imprecisa em termos das representações fonológicas, de modo que a criança pode não identificar claramente todos os fonemas que compõem a palavra que ela quer escrever. Desta forma, ela acaba omitindo a letra ou letras a eles correspondentes. Podem ser citados como exemplos as omissões de certas letras no final de sílabas, cuja construção é consoantevogal-consoante (CVC), sendo que a última consoante ocupa a posição de coda. Omissões de "l", "r" e "s", nesta posição, podem servir de exemplos (churrasco > churraco; estudam > etudam; soltou > 
sotou). Ocorrem também omissões de grupos consonantais, em sílabas consoante-consoante-vogal (CCV) mais especificamente representados com " $r$ " e "l" (presente > pesente). Estes casos parecem ter uma forte influência de uma imprecisão nas representações fonológicas.

Uma das omissões mais freqüentes é a das letras "m" e "n" em final de sílaba consoante-vogalconsoante, posição na qual correspondem a uma marcação da nasalização da vogal e não a uma consoante propriamente dita. Nesta posição a vogal e a consoante (formando am, em, im, om, um e an, en, in, on, un) funcionam como um dígrafo, isto é, duas letras que juntas representam um único fonema. Fica a questão relativa a se os sujeitos não fazem uma diferenciação precisa do aspecto da nasalidade ou, muito provável, não têm informações ou conhecimentos precisos a respeito de como grafar tais fonemas (as vogais nasais), mesmo tendo detectado-o com precisão (manchar > machar; combinar > cobinar). Esta também é a omissão mais comumente encontrada em crianças sem problemas de aprendizagem ${ }^{7}$. Observa-se igualmente a omissão de dígrafos como "qu", "nh" e "lh" também por desconhecimento destas formas mais complexas de representação fonêmica.

O terceiro maior grupo de erros observados diz respeito às palavras grafadas exatamente da maneira como são pronunciadas. Esta situação revela que a criança está realizando uma análise fonética, de acordo com seu modo de pronuncia, identificando os fonemas que compõem a palavra articulada. Esta é a estratégia de correspondência fonema-grafema, que está na base das escritas alfabéticas. As transcrições literais da fala, nestes casos, denotam uma habilidade no nível das representações fonológicas. Porém, como pode haver uma discrepância entre o modo de falar e o de escrever, muitas palavras resultam erradas, caracterizando tal tipo de alteração. A superação deste tipo de erro depende de conhecimentos de natureza ortográfica. Por exemplo, a criança deve compreender que, embora pronuncie a palavra "soltou" com "u" em vez de "I" e sem o "u" final ("soutô"), a convenção ortográfica gera uma discrepância, devendo a palavra ser escrita na forma de "soltou" e não "souto".

Não foram encontrados estudos com análises semelhantes no Português. Porém, comparados com pesquisa anterior sobre a aquisição da ortografia em crianças sem queixas de aprendizagem ${ }^{7}$ verifica-se uma inversão na freqüência de ocorrência dos dois últimos grupos uma vez que as alterações ortográficas por apoio na oralidade tendem a ser mais numerosas que aquelas provocadas por omissão de letras, como foi encontrado neste estudo. Este dado pode ser indicativo de que crianças com dificuldades de aprendizagem podem apresentar limitações maiores em termos da precisão da análise fonológica, resultando em maior número de omissões.

Os demais tipos de erros exibem ocorrências menos freqüentes, e que se assemelham ao perfil encontrado em crianças sem dificuldades ${ }^{7}$. Confirmando a tendência já observada anteriormente ${ }^{7,21}$, os erros relativos à confusão entre letras com traçados semelhantes e as inversões de letras, são aqueles de menor ocorrência, mesmo em crianças com problemas de aprendizagem, de acordo com os dados obtidos neste estudo. Por outro lado, tais resultados contrariam a crença de que os erros chamados de ordem visual, como a dificuldade para perceber a forma das letras e, principalmente a posição das mesmas no espaço ou a respectiva posição na seqüência de letras que compõem as palavras sejam reveladores de transtornos de aprendizagem. Não são déficits de tal natureza que definem os erros de escrita encontrados em crianças com dificuldades de aprendizagem.

A busca de correlações entre erros (Tabelas 4 e 5) mostra que a mais forte dentre elas diz respeito à "omissão de letras" e "outras alterações". Nesta última categoria são classificados erros atípicos, que dada a ocorrência mais restrita, não constituem categorias específicas. Em geral, tais erros revelam falhas predominantemente de natureza fonológica, na forma de substituições decorrentes de representações fonológicas não adequadas, como acontece no caso de algumas substituições de letras (machucado > masucado; girassol > zilasol; manchar > menchar).

Outra das correlações observadas, entre "Junção/separação de palavras" e "representações múltiplas" também parecem evidenciar uma base comum relacionada às representações ortográficas, no caso a noção de palavra, enquanto conjuntos de letras que se separam por espaços. Embora correlações positivas entre "omissão de letras" e "apoio na oralidade" e "apoio na oralidade" e "outras alterações", tenham sido encontradas, não ficaram evidentes, neste trabalho, possíveis bases comuns para tais tipos de erros.

\section{CONCLUSÃO}

De acordo com a amostra obtida, o TDA/H pode ser um dos principais distúrbios apresentados pelas crianças encaminhadas para diagnóstico por apresentarem algum tipo de problema quanto ao rendimento escolar. Quanto ao fluxo de encaminhamentos, que aumenta de acordo com o avançar das séries iniciais, existem evidências de uma demora 
para o diagnóstico, o que pode gerar uma omissão de atendimentos necessários para o perfil de necessidades do aluno com problemas de aprendizagem, agravando o problema e aumentando a defasagem entre desempenho e expectativa escolar.

No que se refere aos tipos de erros encontrados, o maior desafio para os sujeitos apresentando problemas de aprendizagem diz respeito à consolidação das características da escrita regidas por elementos da ortografia, mais do que de problemas de ordem fonológica, embora também estejam muito presentes neste grupo.

Seguindo a ordem determinada pela freqüência média dos erros, pode-se afirmar que, em primeiro lugar aparecem os erros gerados pela complexidade ortográfica relativa à possibilidade de representações múltiplas. Esta característica do português requer certas habilidades, ou seja, que os sujeitos compreendam que um mesmo fonema pode ter várias letras, quais são estes fonemas, quais são estas letras e que estabilize a forma convencional das palavras que apresentam estas possibilidades. Devem também compreender que uma mesma letra pode ter vários sons, quais são tais letras e quais são estes sons. Nestes casos regras contextuais e morfossintáticas desempenham importante papel, devendo ser compreendidas para que os erros ocorram em menor número.

Os erros por omissões, que vêm em segundo lugar, indicam que tais sujeitos ainda não desen- volveram habilidades fonológicas suficientes para garantir a escrita completa das palavras, assim como não dominam completamente certas correspondências entre fonemas e grafemas, o que produz a ausência de algumas letras nas palavras escritas. A existência de dificuldades fonológicas é reforçada pela presença de inúmeros erros classificados como "outras alterações".

A presença também numerosa de erros de escrita por apoio na oralidade indica que os sujeitos ainda não têm suficiente compreensão de que escrever não se limita a uma transcrição fonética e que elementos de natureza ortográfica desempenham um papel determinante na forma de se escrever as palavras.

De modo geral, estes três tipos de erros, que se mostraram mais freqüentes, também são os mais observados em escolares considerados sem problemas de aprendizagem. Este fato indica que, por detrás de tais erros, existem características complexas da língua e que podem ser até mesmo mais desafiadoras para aquelas crianças com dificuldades de aprendizagem.

Por outro lado, os erros considerados de origem visual que levariam a uma confusão entre letras que se assemelham e também à inversão espacial ou trocas na posição das letras dentro das palavras, têm muito baixa freqüência, revelando que capacidades visuais e espaciais não são os fatores que limitam a aprendizagem deste grupo estudado.

\section{ABSTRACT}

Purposes: to analyze orthographic abilities of children diagnosed with some kind of learning problems. Mistakes produced by the subjects were quantified and classified seeking to describe those with higher periodicity, checking if there is any difference among their occurrences. We intended to characterize an indicative spelling profile for the general difficulties that are more commonly found in this population. Methods: the writing of 69 subjects was assessed by the Laboratory of Learning Disturbances of the Neurology Department of UNICAMP and diagnosed as showing some type of learning difficulty. The ages varied between eight years and two months and thirteen years and four months, with a ten year and six months as an average value. Only subjects in alphabetical writing level without any type of intellectual problem were included. The found mistakes were classified in eleven categories and quantified for ends of statistical analysis. Results: ten groups were diagnosed being the Attention Deficit Disorder the most numerous, followed by School Difficulties and Associated Disorders. Most of the subjects studied the third grade of the elementary school. The most recurrent type of spelling mistake was "Multiple Representations", followed by "Omissions" and "Support in Orality". Significant correlation was mainly noted among "Omissions" and "Other Alterations". Conclusion: the major difficulty found is centered in the "Multiple Representations", mainly revealing difficulties at the orthographic level. The mistakes for "Letter Omission" arise from flaws in the process of phonological analysis and phoneme-grapheme correspondence. The low occurrence of mistakes for "Inversion" and "Similar Letters", indicates absence of difficulties regarding visual-space nature.

KEYWORDS: Learning Disorders; Dyslexia; Attention Deficit Disorder with Hyperactivity 


\section{REFERÊNCIAS}

1. Caravolas M, Volín J. Phonological spelling errors among dyslexic children learning a transparent orthography: the case of Czech. Dyslexia. 2001; 7(4):229-45.

2. Romonath R, Wahn C, Gregg N. [Phonological and orthographic processes of reading and spelling in young adolescents and adults with and without dyslexia in German and English: impact on foreign language learning]. Folia Phoniatr Logop. 2005; 57(2):96-110.

3. Juul H, Sigurdsson B. Orthography as a handicap? A direct comparison of spelling acquisition in Danish and Icelandic. Scand J Psychol. 2005; 46(3):263-72.

4. Ziegler JC, Goswami U. Reading acquisition, developmental dyslexia, and skilled reading across languages: a psycholinguistic grain size theory. Psychol Bull. 2005; 131(1):3-29.

5. Seymour PH, Aro M, Erskine JM. Foundation literacy acquisition in European orthographies. $\mathrm{Br} \mathrm{J}$ Psychol. 2003; 94(Pt 2):143-74.

6. Carraher T. Explorações sobre o desenvolvimento da ortografia em português. Secretaria da educação: coordenadoria de estudos e normas pedagógicas. Isto se aprende com o ciclo básico. São Paulo: SE/ CENP; 1990. p.114-22.

7. Zorzi JL. Aprender a escrever: a apropriação do sistema ortográfico. Porto Alegre: Artmed; 1998. $115 p$.

8. Morais AG. Ortografia: este espetacular objeto de conhecimento. In: Morais AG, organizador. O aprendizado da ortografia. Belo Horizonte: Autêntica; 2000. p.7-19.

9. Rego LLB, Buarque LL. Algumas fontes de dificuldade na aprendizagem de regras ortográficas. In: Morais AG, organizador. O aprendizado da ortografia. Belo Horizonte: Autêntica; 2000. p. 21-41.

10.Zorzi JL. Aprendizageme distúrbios da linguagem escrita. Porto Alegre: Artmed; 2003. 174p.

11. Zanella MSZ. Leitura e aprendizagem da ortografia: um estudo com alunos de $4^{\underline{a}}$ a $6^{\underline{a}}$ série do Ensino Fundamental [tese]. São Paulo (SP): Pontifícia Universidade Católica; 2007.

12. Grigalevicius MM. Aprendizagem da linguagem escrita: um estudo sobre a competência ortográfica de alunos da $5^{\text {a }}$ série do ensino fundamental [dissertação]. São Paulo (SP): Pontifícia Universidade Católica; 2007.

13. Santos TMS. Vocabulário, consciência fonológica e nomeação rápida: contribuições para a ortografia e elaboração escrita. [tese]. São Paulo (SP): Universidade de São Paulo; 2007.
14. Valente, HMR. Análise da onda da fala com crianças com alterações na escrita quanto ao traço de sonoridade [dissertação]. São Paulo (SP): Pontifícia Universidade Católica; 1997.

15. Osmon DC, Braun MM, Plambeck EA. Processing abilities associated with phonologic and orthographic skills in adult learning disability. J Clin Exp Neuropsychol. 2005; 27(5):44-54.

16. Connelly V, Campbell S, MacLean M, Barnes J. Contribution of lower order skills to the written composition of college students with and without dyslexia. Dev Neuropsychol. 2006; 29(1):175-96.

17. Silliman ER, Bahr RH, Peters ML. Spelling patterns in preadolescents with atypical language skills: phonological, morphological, and orthographic factors. Dev Neuropsychol. 2006; 29(1):93-123.

18. Ciasca SM, Moura-Ribeiro MVL. Avaliação e manejo neuropsicológico da dislexia. In: Rotta NT, Ohlweiler L, Riesgo RS. Transtornos da aprendizagem: abordagem neurobiológica e multidisciplinar. Porto Alegre: Artmed; 2006. p.181-94.

19. Ciasca SM. Distúrbios de aprendizagem e transtornos da atenção: algumas reflexões. In: Maluf Ml, organizador. Tramas do conhecimento, do saber e da subjetividade. Petrópolis: Vozes; 2006. p.237-44.

20. Zorzi JL. Os distúrbios de aprendizagem e os distúrbios específicos de leitura e da escrita. In: Britto ATBO, organizador. Livro de fonoaudiologia. São José dos Campos: Pulso Editorial; 2005. p. 217-30.

21. Zorzi JL. Alterações ortográficas nos transtornos de aprendizagem. In: Maluf MI, organizador. Tramas do conhecimento, do saber e da subjetividade. Petrópolis: Vozes; 2006. p.144-62.

22. Gustafson S, Ferreira J, Rönnberg J. Phonological or orthographic training for children with phonological or orthographic decoding deficits. Dyslexia. 2007; 13(3):211-29.

23. Goswami U. Phonological representations, reading development and dyslexia: towards a crosslinguistic theoretical framework. Dyslexia. 2000; 6(2):133-145.

24. Spencer K. Is English a dyslexic language? Dyslexia. 2000; 6(2):152-62.

25. Spencer K. Differential effects of orthographic transparency on dyslexia: word reading difficulty for common English words. Dyslexia. 2001; 7(4):217-28.

26. Capovilla AGS, Joly MCRA, Ferracini F, Caparrotti NB, Carvalho MR, Raad AJ. Estratégias de leitura e desempenho em escrita no início da alfabetização. Psicol Esc Educ. 2004; 8(2):189-97. 27. Meireles ES, Correa J. Regras contextuais e morfossintáticas na aquisição da ortografia da 
língua portuguesa. Psicologia: Teoria e Pesquisa. 2005; 21(1):77-84.

28. Ciasca SM. Avaliação neuropsicológica e neuroimagem nos distúrbios de aprendizagem, leitura e escrita. In: Associação Brasileira de Dislexia. Dislexia: cérebro, cognição e aprendizagem. São Paulo: Frontis; 2000. p.127-33.

29. Shaywitz SE, Shaywitz BA. Dyslexia (specific reading disability). Biol Psychiatry. 2005; 57(11):1301-9.

30. Ferreiro E, Teberosky A. Psicogênese da língua escrita. Porto Alegre: Artes Médicas; 1986. 284p.

31. Olofsson A. Naming speed, phonological awareness and the initial stage of learning to read. Logop Phoniatr Vocol. 2000; 25(1):35-40.
32. Zorzi JL. A inversão de letras na escrita: o fantasma do espelhamento. Pró-Fono. 2001; 13(2):212-8.

33. Bourassa D, Treiman R. Spelling in children with dyslexia: analyses from the Treiman-Bourassa. Sci Stud Read. 2003; 7(4):309-33.

34. Cao F, Bitan T, Chou T, Burman D, Booth J. Deficient orthographic and phonological representations in children with dyslexia revealed by brain activation patterns. J Child Psychol Psych. 2006; 47(10):1041-50.

35. Mackie C, Dockrell JE. The nature of written language deficits in children with SLI. J Speech Lang Hear Res. 2004; 47(6):1469-83.

RECEBIDO EM: 01/05/2008

ACEITO EM: 10/08/2008

Endereço para correspondência:

Rua Cayowaá, 664

São Paulo - SP

CEP: 05018-000

Tel/Fax: (11) 3868-0818

E-mail: jaime@cefac.br 